\title{
Correlation of Wells Score, Caprine Score, and Padua Score with Risk of Hypercoagulation Condition Based on D-dimer in Intra-articular, Periarticular, and Degenerative Fracture Patients of Inferior Extremity
}

\author{
Thomas Erwin Christian Junus Huwae* (D), Ahmad Heifan, Muhammad Alwy Sugiarto (D) \\ Department of Orthopaedics and Traumatology, Faculty of Medicine, Universitas Brawijaya, RSUD Dr. Saiful Anwar, Malang, \\ Indonesia
}

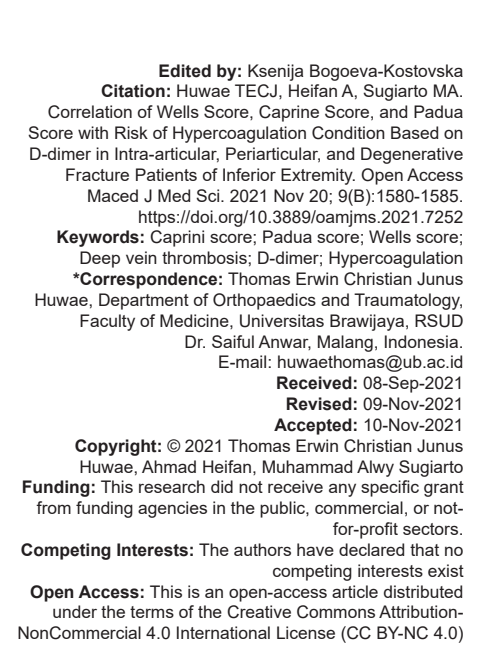

\begin{abstract}
BACKGROUND: Surgery for large joint areas can increase risk of venous thromboembolism, which can be in the form of pulmonary embolism or deep vein thrombosis (DVT). As much as, $40-60 \%$ of hemostasis abnormalities specifically hypercoagulable diseases, are suspected of causing this condition. The risk of developing DVT can be assessed using a physiological examination such as Wells score, Caprini score, and Padua score. The scoring systems assess some of the patient's symptoms and risk factors for increasing the incidence of DVT. Hypercoagulation conditions can be assessed using D-dimer, which is often considered a gold standard in measuring hypercoagulation conditions or as an indicator of DVT.

AIM: We aimed to investigate correlation of Wells Score, Caprine Score, and Padua Score with risk of hypercoagulation condition based on d-dimer in intra-articular, periarticular, and degenerative fracture patients of inferior extremity.

METHODS: This study used a cross-sectional design and was conducted on 34 participants that undergoing periarticular surgery. This study compared the Wells, Caprini, and Padua scores test against hypercoagulation conditions confirmed by the D-dimer examination.

RESULTS: The correlation between Wells, Padua, Caprini scores, and D-dimer was 0.676, 0.023, and 0.395 respectively.

CONCLUSION: There was a significant relationship between the Padua scores and the D-dimer.
\end{abstract}

\section{Introduction}

Injuries to the extremities can lead to fractures and dislocations. The overall fracture incidence is 11.3 in $1000 /$ year over 30 years old. The incidence of fracture in men is higher than in women, 11.67 in 1000 every year. However, incidents in some parts of the world will be different [1]. According to a study conducted in seven Asian countries, large joint surgery can increase the risk of venous thromboembolism (pulmonary embolism or deep vein thrombosis [DVT]) by 40-60\% [2]. Meanwhile, another research conducted showed that the incidence of DVT in Asian people after joint replacement surgery ranged from $48.1 \%$ to $67.7 \%$ [3].

DVT is suspected to happen in post-operative patients in the joint area due to patients with hemostasis problems who are hypercoagulable. The standard examination to determine DVT is to use venography [4]. However, these examinations are not available in all health facilities in a developing country, especially in
Indonesia. Therefore, another diagnostic instrument is needed to diagnose DVT [5]. Several scoring systems such as Wells, Padua, and Caprini were developed to assess a person's risk for DVT. The scoring system is an easy and non-invasive way to determine hypercoagulable conditions, one of the causes of DVT. Although, there has been little discussion about correlation between scoring method with D-dimer on the patient with the high risk for DVT. In this case, patients undergoing periarticular surgery have a high risk of DVT incidence. The scoring system assesses several symptoms and risk factors in patients who are developing at the high risk of DVT incidence [4]. This study set out to investigate the usefulness of a scoring system as a practical and safe method and can be used in all health-care facilities, even though in the primary health-care facility [5], [6].

Since there is a high correlation between coagulation status and the risk of DVT, assessing coagulation status can be helpful to determine the risk of DVT. One commonly used test is the measurement 
of D-dimer levels, which is often used as a gold standard in measuring hypercoagulable conditions or as an indicator of fibrinolysis [7]. A positive test result shows the existence of a thrombus, but it does not specify the abnormality or rule out some other causes. The D-dimer examination is helpful to determine the formation of abnormal blood clots or the presence of thrombotic events (indirect) and clot lysis or fibrinolytic processes (direct) [8].

\section{Methods}

This study was conducted at the Department of Orthopedic Surgery, Central Laboratory Installation, RSUD dr. Saiful Anwar Malang during the 2020 period with subjects consisting of periarticular surgery patients at RSUD Dr. Saiful Anwar Malang who met the inclusion and exclusion criteria that had been set. This study consisted of independent variables in the form of Wells, Padua, and Caprini scores. Meanwhile, the dependent variable in this study was the hypercoagulable condition.

\section{Study design}

This study used a cross-sectional design and compared the Wells, Caprini, and Padua scores against hypercoagulable conditions confirmed by the D-dimer examination. The output of the diagnostic test was the correlation value as the significance of each variable between Well, Caprini, and Padua score with D-dimer level.

\section{Research subjects}

This study was conducted on patients undergoing periarticular surgery at RSUD dr. Saiful Anwar Malang has met the inclusion and exclusion criteria set during the 2020 period.

The inclusion criteria in this research subjects must be patients aged over 40 years who were scheduled to undergo intra-articular and periarticular fracture surgery due to trauma or degenerative lower extremity, not consuming coagulant at least 5 days before surgery, and willing to participate in the study. The diagnosis of DVT was made by a doctor based on anamnesis, physical examination, and Doppler ultrasound. Exclusion criteria in this study included age below 40-years-old, patients with multiple trauma, patients with a moderate or severe head injury, patients who had varicose veins before surgery, patients with severe hepatic dysfunction, patients with severe renal impairment, patients with a history of hemostatic disorders, and patients with pre-operative DVT.
Patients who underwent surgery were chosen for this study because they had venous stasis, vascular damage, or hypercoagulability post-surgery. Any of these three factors can trigger DVT.

\section{Sampling}

The sample size obtained was 34 based on patient who met the inclusion criteria (Figure 1). We obtain minimum sample size formula to determine the correlation or specificity of examination, and AUC is described as:

$$
\begin{aligned}
& n=\frac{Z_{a}^{2} \cdot p(1-p)}{e^{2}} \\
& Z_{a}^{2}=\text { Confidence level at } 95 \% \text { (Standard }
\end{aligned}
$$
value of 1.96)

$$
\begin{aligned}
& p=\text { Variance of population } 50 \%(0.5) \\
& e=\text { Allowable error }(20 \%) .
\end{aligned}
$$

\section{Data collection and analysis}

Primary data in the form of physiological hemostasis profile were obtained from the D-dimer condition, and this was used in this research to estimate the risk of thromboembolism, which can predict DVT.

The data collection techniques used are secondary data in the form of patient characteristics, including gender, age, weight, height, and other DVT risk factors contained in three scoring systems carried out by physical examination and interviews by researchers in the data collection period.

The data that have been collected will then be analyzed. Data analysis was carried out to determine

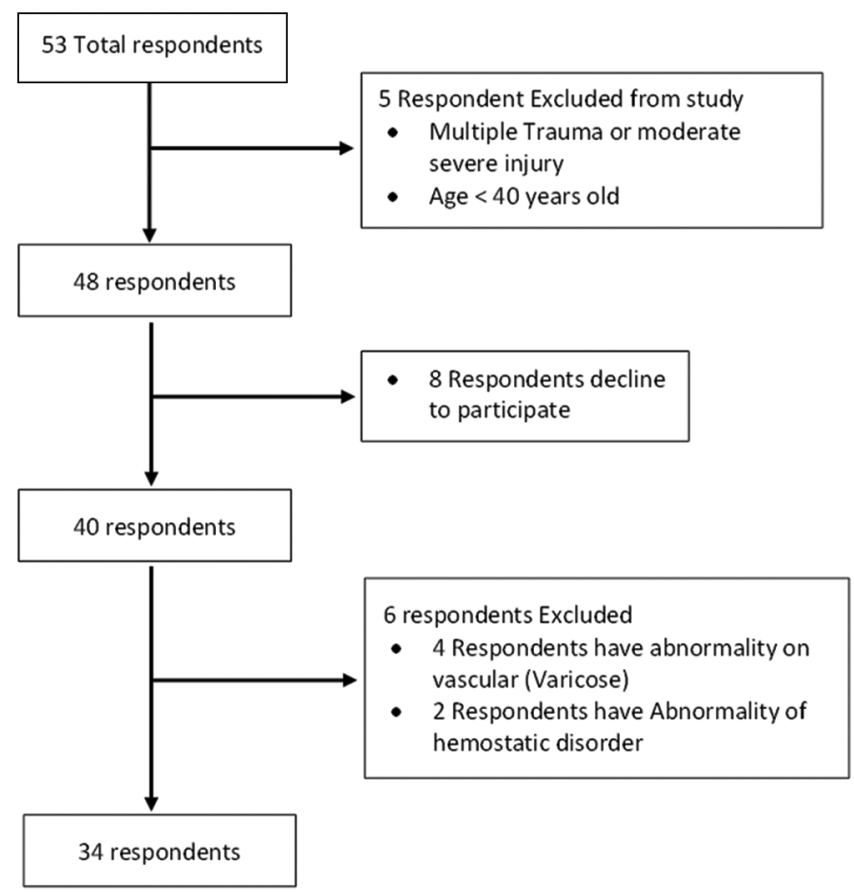

Figure 1: Flow chart patient inclusion 
whether there was a relationship between the Wells, Padua, and Caprini scores with D-dimer using the Pearson correlation statistical test. However, first, we should test the normality assumption in the data using the Kolmogorov-Smirnov test. If the data are not normally distributed, the test is carried out using the Spearman correlation test. The statistical test was carried out with SPSS software with a confidential level was $95 \%$ or an error rate was $5 \%$.

\section{Results}

\section{Research subjects characteristics}

There are two types of primary and secondary data. Gender, age, weight, height, and D-dimer of respondents are the primary data, while Wells score, Padua score, and Caprini score are the secondary data. BMI score in this study according BMI Asia pacific classification. Table 1 shows research subject characteristics and DVT scores characteristics.

Table 1: Research subject characteristics and DVT scores characteristics

\begin{tabular}{llll}
\hline Characteristic & & Frequency & Percentage \\
\hline Gender & & 14 & \\
Male & & 20 & 41.2 \\
$\quad$ Female & & 6 & 58.8 \\
Age & & 21 & \\
$\quad<50$ & 7 & 17.6 \\
$51-80$ & & 2 & 20.8 \\
$>80$ & & 27 & \\
BMI & & 4 & 5.9 \\
$\quad$ Underweight & & 1 & 79.4 \\
Normal & & Frequency & 32.4 \\
Overweight & & 29 & 8.8 \\
Obese & & 4 & 100 \\
$\quad$ Total & Total score & 1 & 85.3 \\
\hline DVT score & 0 & 34 & 11.8 \\
\hline Wells score & 1 & 4 & 2.9 \\
& 2 & 12 & 100 \\
Total & 0 & 18 & 11.8 \\
Padua score & 1 & 34 & 35.3 \\
& 2 & 0 & 52.9 \\
Total & & 1 & 100 \\
Caprini score & $0-1$ & 27 & 0 \\
& 2 & 6 & 2.94 \\
Total & $3-9$ & 34 & 79.4 \\
\hline DVT: & $>9$ & & 17.6 \\
\hline
\end{tabular}

DVT: Deep vein thrombosis, BMI: Body mass index.

\section{D-Dimer characteristic}

The D-dimer value was in the range 0.17-9.44 mg/L and a mean of $3.028 \mathrm{mg} / \mathrm{L}$ with SD 2.055 .

\section{Correlation between Wells score, Padua} score, and Caprini score with D-dimer

Analysis Pearson correlation was performed to determine the relationship between Wells, Padua, and Caprini scores with D-dimer.
Based on the normality test results using Kolmogorov-Smirnov, it can be seen that the Wells score and Padua score data were not normally distributed because they have a significance value of $<0.05$, and further analysis can be carried out using Spearman correlation. On the other hand, while the Caprini score and D-dimer have a significance value of $>0.05$, the data were normally distributed, and further analysis can be done using Pearson correlation.

Based on Table 2, it can be seen that only the Padua correlation score with D-dimer has a significant relationship with $p<0.05$.

Table 2: Correlation analysis of Wells, Padua, and Caprini scores with D-dimer

\begin{tabular}{ll}
\hline DVT score & D-dimer \\
\hline Wells score & \\
P & 0.676 \\
N & 34 \\
Padua score & \\
P & 0.023 \\
N & 34 \\
Caprini score & \\
P & 0.395 \\
N & 34 \\
\hline DVT: Deep vein thrombosis &
\end{tabular}

\section{Discussion}

\section{D-dimer \\ Correlation between Wells score and}

Wells score consists of assessment ten criteria. This scoring system is changed or modified for DVT probability by taking one of the other clinical features, that is, patients with a previous history of DVT given an additional one point. Based on the modified Wells score [9], Wells score is a scoring system to assess a person's risk of developing DVT that is consist of a history of active cancer (undergoing treatment in the past 6 months), calf swelling $>3 \mathrm{~cm}$, superficial venous dilatation, pitting edema, the previous history of DVT, swelling of the entire leg, tenderness along the venous system, paralysis or application of casts to the lower extremities, bed rest $>3$ days, and a history of the major surgery requiring regional or general anesthesia in the past 12 weeks, that in each point has +1 point. Therefore, alternative diagnosis is at least as likely as DVT point -2 point. Eventually, the outcome is calculated by adding all of the points with conclusion DVT likely two points or more, and one point or less unlikely DVT. From Table 1, the results of the descriptive analysis show that the majority of respondents have a Wells score $<1$ point, 29 respondents or $85.3 \%$ of the total respondents, which indicate the majority of respondents have a low risk of DVT.

Furthermore, the results of the descriptive analysis of D-dimer showed that the average value of D-dimer was 3.028 , where this value was above the 
value of D-dimer for the normal category. This typical condition indicates that the average respondent had a high risk of developing a DVT. D-dimer was a blood sample test to diagnose diseases and conditions that cause hypercoagulation (a tendency for blood to clot beyond its normal size). The D-dimer test was used to monitor and keep blood clotting levels from increasing so that doctors could use the D-dimer test to rule out DVT. The higher the value of the D-dimer, the stronger the suspicion of a blood clot. It was as stated by Kosinski (2004) that D-dimer levels that were higher than the reference normal value indicate the presence of the high levels of fibrin degradation products and means that there was a formation and breakdown process of thrombus in the body [10].

However, from correlation analysis showed that there was no significant relationship between the Wells score and D-dimer because the majority of respondents studied based on the results of the Wells score have a low risk of developing DVT, which was supported by the results of the analysis of the D-dimer test, which showed that the average respondent was included in the normal category. Research by Alasiry et al. found that the Wells score was associated with D-dimer levels and the results of Doppler ultrasound. However, only patients with moderate and high Wells scores were included in the analysis in that study. Unfortunately, in this study, there were no samples with moderate or high scores [11].

\section{D-dimer}

\section{The correlation between Padua score and}

Meanwhile, the Padua score consists of 11 risk categories. This score is generally used to predict the presence of DVT in patients with cancer as the primary diagnosis [12]. The study conducted by Nendaz et al. obtained the sensitivity and specificity of the Padua scoring of $73.3 \%$ and $51.9 \%$, respectively [13].

The Padua score consists of active cancer, the previous VTE (with exclusion of superficial vein thrombosis), reduced mobility, and already known thrombophilic condition, which has three points, respectively. Then, two points for recent $(\leq 1 \mathrm{mo})$ trauma dan/or surgery, and one point for elderly age ( $\geq 70$ years), hearth and/or respiratory failure, acute myocardial infarction or ischemic stroke, acute infection and/or rheumatologic disorder, obesity (BMI $\geq 30$ ), and ongoing hormonal treatment. This score has a conclusion that the low risk of DVT is defined as one of $<4$ and high risk is defined as a cumulative score $\geq 4$ [14].

The Padua score is the scoring options that are often used for assess the patients' quality of thrombus formation risk. A thrombus is the formation of a blood clot, known as DVT, if it occurs in a vein [14]. The results of the descriptive analysis showed that if all of the respondents have a Padua score between 0 and 2 where the value was still below the value of 4 , which indicates that all respondents have a low risk for DVT. These results might be due because the research design was used a cross-sectional design. Further research can use a case-control design so that it can be compared between samples that have a high and low risk of DVT.

Based on the Spearman correlation analysis results, it was known that the Padua score has a significant relationship with D-dimer. There was a significant relationship between Padua score and D-dimer because both D-dimer and Padua score predictors thromboembolism. Research by Barbar et al. (2010) concluded that the Padua score has a good potential predictor of thromboembolic risk compared with common practice [15]. It correlated with study by Zhang et al. (2020). The combination of a Padua prediction score $\geq 4$, D-dimer $>1.0 \mu \mathrm{g} / \mathrm{mL}$, and CURB65 score has a sensitivity of $88.52 \%$ and a specificity of $61.43 \%$ for screening for DVT [16].

\section{The correlation between Caprini score and}

\section{D-dimer}

Another scoring system is the Caprini score, which assesses 40 risk factors for DVT occurrence, each with its score burden. Patients with a Caprini score $>9$ have a high risk of developing DVT. The Caprini score consists of several groups of risk factors, each of which has its weight rating with the following interpretation: A score of $0-1$ indicates a low risk of DVT (<10\%). A score of 2 indicates a moderate risk of developing DVT (10-20\%). A score of 3-9 indicates a high risk of DVT $(20-40 \%)$. A score $>9$ indicates a very high risk of DVT (40-60\%).

Caprini score is a scoring system that aims to assess risk factors associated with an increased risk of developing a DVT. The descriptive analysis of the Caprini score showed that the most respondents have a Caprini score between 3 and 9, which was $79.4 \%$ of the total respondents and indicates that the most respondents have a high risk of DVT. The advantage of the Caprini score is that it was developed for use in surgical patients. However, the previous studies have shown that the cutoff value of the Caprini score is low enough so that it is difficult to predict which patients need anticoagulation or not [6]. This was also supported by the results of this study, where the assessment using the Wells and Padua scores showed that the most patients were at low risk, in contrast to the assessment results using the Caprini score.

Wirabhawa et al. (2020), in their study, stated that the Caprini score was related to the value of D-dimer, contrary to the results of this study. To better describe the relationship of this scoring system with hypercoagulation conditions, further research can use a case-control or retrospective cohort design [17].

This research had some limitations in its implementation. First, the design of this study was 
cross-sectional, which can only see whether there is a relationship between Wells, Caprini, and Padua scores with temporary hypercoagulable conditions. To see a better relationship between these two variables, conducting a retrospective study such as a casecontrol or retrospective cohort is necessary. Second, the sampling system of this study uses a consecutive sampling system with a sample of 34 people. Therefore, a future study with a more significant number of samples is required to generate more representative results.

\section{Conclusion}

There was a significant relationship between the Padua score with the D-dimer. On the other hand, the results for the other variables are not significant. Hence, Padua score can be helpful as a predictor of the condition hypercoagulation of DVT.

\section{Ethical Approval}

The Ethics Committee of Syaiful Anwar General Hospital with number 400/088/K.3/302/2020 has approved all protocols were carried out according to the relevant guidelines and regulations.

\section{Author's Contribution}

Study Design: Thomas E. C. J. Huwae and Ahmad Heifan. Data Collection: Thomas E. C. J. Huwae and Ahmad Heifan. Statistical Analysis: Thomas E. C. J. Huwae and Muhammad Alwy Sugiarto. Data Interpretation: Thomas E. C. J. Huwae and Ahmad Heifan. Manuscript Preparation: Ahmad Heifan and Muhammad Alwy Sugiarto. Literature Search: Ahmad Heifan and Muhammad Alwy Sugiarto. Funds Collection: This research did not receive any specific grant from funding agencies in the public, commercial, or not-for-profit sectors.

\section{References}

1. Bohl DD, Maltenfort MG, Huang R, Parvizi J, Lieberman JR, Della Valle CJ. Development and validation of a risk stratification system for pulmonary embolism after elective primary total joint arthroplasty. J Arthroplasty. 2016;31(9):187-91. https://doi. org/10.1016/j.arth.2016.02.080

\section{PMid:27067463}

2. Piovella F, Wang CJ, Lu H, Lee K, Lee LH, Lee WC, et al Deep-vein thrombosis rates after major orthopedic surgery in Asia. An epidemiological study based on postoperative screening with centrally adjudicated bilateral venography. J Thromb Haemost. 2005;3(12):2664-70. https://doi. org/10.1111/j.1538-7836.2005.01621.x

PMid: 16359505

3. Flevas DA, Megaloikonomos PD, Dimopoulos L, Mitsiokapa E, Koulouvaris $\mathrm{P}$, Mavrogenis AF. Thromboembolism prophylaxis in orthopaedics: An update. EFORT Open Rev. 2018;3(4):130-42. https://doi.org/10.1302/2058-5241.3.170018

PMid:29780621

4. Tovey C, Wyatt S. Diagnosis, investigation, and management of deep vein thrombosis. BMJ. 2003;326(7400):1180-4. https://doi. org/10.1136/bmj.326.7400.1180

PMid: 12775619

5. Gatot D, Mardia Al. Differences of wells scores accuracy, caprini scores and Padua scores in deep vein thrombosis diagnosis. IOP Conf Ser Earth Environ Sci. 2018;125(1):012131. doi:10.1088/1755-1315/125/1/012131

6. Grant PJ, Greene MT, Chopra V, Bernstein SJ, Hofer TP, Flanders SA. Assessing the caprini score for risk assessment of venous thromboembolism in hospitalized medical patients. Am J Med. 2017;129(5):528-35. https://doi.org/10.1016/j. amjmed.2015.10.027

PMid:26551977

7. Bateman DK, Dow RW, Brzezinski A, Bar-Eli HY, Kayiaros ST Correlation of the caprini score and venous thromboembolism incidence following primary total joint arthroplasty-results of a single-institution protocol. J Arthroplasty. 2017;32(12):3735-41. https://doi.org/10.1016/j.arth.2017.06.042

PMid:28734614

8. Barber M, Langhorne P, Rumley A, Lowe GD, Stott DJ. D-dimer predicts early clinical progression in ischemic stroke: Confirmation using routine clinical assays. Stroke. 2006;37(4):1113-5. https:// doi.org/10.1161/01.STR.0000209240.63821.1a PMid:16527998

9. Nanavati N, Hoggett L, Hampton M, Gordon A. Venous thromboembolism after orthopaedic surgery-how long is the patient at risk? J Ageing Res Healthc. 2016;1(2):12-20. https:// doi.org/10.14302/issn.2474-7785.jarh-16-1067

10. Kosinski CM, Mull M, Schwarz M, Koch B, Biniek R, Schläfer J, et al. Do normal D-dimer levels reliably exclude cerebral sinus thrombosis? Stroke. 2004;35(12):2820-5. https://doi. org/10.1161/01.STR.0000147045.71923.18 PMid:15514174

11. Alasiry E, Benyamin AF, Saleh S, Bakri S, Ilyas M, Kasim H, et al. Correlation of wells score, prothrombin time, activated partial thromboplastin time, fibrinogen and D-dimer levels with Doppler ultrasonography in suspected deep vein thrombosis patients. Glob J Health Sci. 2020;12(11):1-6.

12. Chaudhary R, Damluji A, Batukbhai B, Sanchez M, Feng E, Chandra Serharan $M$, et al. Venous thromboembolism prophylaxis: Inadequate and overprophylaxis when comparing perceived versus calculated risk. Mayo Clin Proc Innov Qual Outcomes. 2017;1(3):242-7. https://doi.org/10.1016/j. mayocpiqo.2017.10.003

PMid:30225423

13. Nendaz M, Spirk D, Kucher N, Aujesky D, Hayoz D, Beer JH, et al Multicentre validation of the Geneva risk score for hospitalised medical patients at risk of venous thromboembolism. Explicit assessment of thromboembolic risk and prophylaxis for medical patients in Switzerland (ESTIMATE). Thromb Haemost. 2014;111(3):531-8. https://doi.org/10.1160/TH13-05-0427 PMid:24226257 
14. Zhou H, Hu Y, Li X, Wang L, Wang M, Xiao J, et al. Assessment of the risk of venous thromboembolism in medical inpatients using the Padua prediction score and caprini risk assessment model. J Atheroscler Thromb. 2018;25(11):1091-104. https:// doi.org/10.5551/jat.43653

PMid:29540637

15. Barbar S, Noventa F, Rossetto V, Ferrari A, Brandolin B, Perlati M, et al. A risk assessment model for the identification of hospitalized medical patients at risk for venous thromboembolism: The Padua prediction score. J Thromb Haemost. 2010;8(11):2450-7. https://doi.org/10.1111/j.1538-7836.2010.04044.x

PMid:20738765
16. Zhang L, Feng $\mathrm{X}$, Zhang $\mathrm{D}$, Jiang $\mathrm{C}$, Mei $\mathrm{H}$, Wang J, et al Deep vein thrombosis in hospitalized patients with COVID-19 in Wuhan, China: Prevalence, risk factors, and outcome. Circulation. 2020;142(2):114-128. https://doi.org/10.1161/ CIRCULATIONAHA.120.046702

PMid:32421381

17. Wirabhawa M, Kawiyana KS, Suyasa IK, Astawa P, Ridia KG Dusak IW, et al. relationship and cut-off point of the Caprini score on D-dimer as a risk parameter for deep vein thrombosis in patients after internal fixation of long bone fractures of the lower extremities. Dig Med Sci. 2020;11(1):291. https://doi. org/10.15562/ism.v11i1.555 